The fluid-blood level in intracranial haematoma due to anticoagulant medication

Sir: The occurrence of intracranial haemorrhage in patients receiving anticoagulant medication is a well-documented complication. ${ }^{1}$ This complication most commonly occurs in patients with poorly controlled haemostasis. Intracranial haemorrhage may also occur in anticoagulated patients with carefully controlled haemostasis as manifested by coagulation results (bleeding time, prothrombin time, partial thromboplastin time) which are within the accepted therapeutic range. I wish to describe the fluid-blood level in five anticoagulated patients with intracranial haemorrhages.

A typical case history is that of a 57 year old normotensive woman with a history of two prior myocardial infarctions and was admitted to hospital because of substernal chest pain. Electrocardiogram was consistent with new acute ischaemic changes. Her blood pressure was $130 / 70 \mathrm{~mm} \mathrm{Hg}$. She was neurologically normal. She was treated with a loading dose of intravenously administered streptokinase and heparin. Her prothrombin time (180 seconds with normal of 20 seconds) and partial thromboplastin time (180 seconds with normal of 50 seconds) became markedly elevated. The dose of streptokinase was reduced and the heparin was discontinued. Despite this, 4 hours later she suddenly became unresponsive, right hemiplegic, apnoeic with no response to noxious stimulation, negative dolls head and negative caloric response, fixed and dilated pupils. CT was immediately performed and showed a large left hemisphere intracerebral haemorrhage with a fluid-blood level (fig). The patient died 4 hours later and no necropsy was performed.

In patients with normal haemostasis who develop an intracranial haemorrhage, CT shows a homogeneously nonenhancing hyperdense lesion in the acute stage. In certain anticoagulated patients, the appearance of the acute haematoma may be different, with the finding of a fluid-blood interface which is unique for this condition. ${ }^{2}$ It is believed that the fluid seen above the haematoma represents plasma; the layering is a sedimentation effect. This appearance of layering within the haematoma was seen in all our patients. This fluid-blood interface occurred when there was not adequate hemostasis. When anticoagulant medication was discontinued and coagulation studies returned to normal, CT showed hyperdense hematoma without the fluid-blood interface. This change was seen in two of these patients.

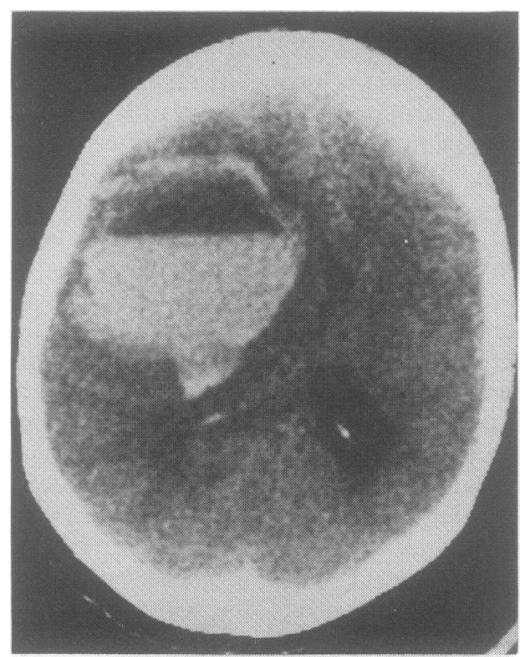

References

1 Snyder M, Renaudin J. Intracranial haemo rhage associated with anticoagulation the apy. Surg Neurology 1977;7:31-4.

2 Livoni JP, McGahan JP. Intracran fluid-blood levels in the anticoagulated patient. Neuroradiology 1983;25:335-7. of

3 Dublin AB, Norman D. Fluid-blood leve in cystic cerebral metastatic melanoma J Comp Assist Tomogr 1979;3:650-2.

4 Richmond T, Virapongse C, Sarwar M. Intra parenchymal blood-fluid levels: New GI sign of arteriovenous malformation rupture. Am J Neuroradiol 1981;2:577-9.

5 Zilkha A. Intraparenchymal fluid-blood lev영 A CT sign of recent intracerebral haemot rhage. J Comp Assisted Tomogr 1983; 301-3.

Accepted 23 December 1986

Fig CT shows a left hemispheric hyperdense lesion with a sharply demarcated fluid-blood level.

The finding of intracranial fluid-blood interface has been seen in patients with peritumoural haemorrhage ${ }^{3}$ and ruptured arteriovenous malformations. ${ }^{4}$ In patients with angiomas, the origin of the fluid-blood level is believed to be haemorrhage into cystic spaces. The fluid-blood level has been reported with primary (glioblastoma multiforme) and metastatic neoplasms (melanoma, lung). In neoplastic conditions in which a fluid-blood interface is seen, there is frequently enhancement at the lesion periphery. In one report, fluid-blood level was seen in patients with spontaneous intracerebral haematoma without any identified aetiology or abnormal haemostasis. ${ }^{5}$ In these cases several potential mechanisms for fluid-blood level were postulated; (1) haemorrhage was acute and clotting of extravasated blood had not been completed when CT performed, (2) large haematoma occurs within the brain using up all clotting factors to result in two components and create fluid-blood interface. If either of these mechanisms were responsible for the fluid-blood interface, we would have expected this finding of fluidblood interface to be common in other intracerebral haematomas. We have not seen this finding in nontumorous intracerebral haemorrhage unless there was impaired haemostasis, as seen in these five patients.

LEON A WEISBERG

Tulane Medical School,

Department of Neurology and Psychiatry. 1430 Tulane Avenue,

New Orleans, Louisiana 70112

$U S A$
Reversible movement disorder in a patient with post traumatic basal ganglia haematoma Sir: The eponyms Brueghel's syndrome ${ }^{1}$ ถูำ Meige syndrome or disease ${ }^{2}$ have beew coined for an idiopathic disorder consiseng of blephrospasm and/or oromandibutar dystonia of adult onset. The symptoms are progressive or static, though improvenef in a few cases has been reported. ${ }^{34}$ th cause of Meige disease is not known andôेंdences for its organic nature are indirect A single report on the brain pathology dice not reveal any abnormality. ${ }^{5}$ Forno 6 described nerve cell loss and other reactions of the substantia nigra in a brain with an ol basal ganglia infarct. We describe a patien $\bar{\phi}$ who developed transient oromandibulat dystonia with blephrospasm following basi ganglia hematoma presumably of post traumatic origin.

A 64 year old man had an accidental faî̉ and sustained an abrasion over left temporal region and a left periorbital haematoma. He. was unconscious for 15 minutes and there after complained of a dull continuous head ache with inability to concentrateSensorium deteriorated again over next 3 days. He was reported to be irritable, consfused and drowsy. He was admitted to provincial hospital where involuntary eye closure, clenching of teeth, forceful jaw opening and jerking of limbs were noted bilaterally, but more on right side. He wa unable to walk owing to unsteadiness involuntary posturing and stiffness of bodyer A left carotid angiogram was performed and 
reported as normal. Hyponatraemia (serum sodium $113 \mathrm{mmol} / \mathrm{l}$ ) and hypokalaemia (serum potassium $2.4 \mathrm{mmol} / \mathrm{l}$ ) were noted. Correction of electrolyte depletion was accompanied by improvement in orientation and alertness over the next week. During this period he was found to be passing 4-51 of dilute urine (specific gravity 1005 to 1008) per 24 hours. In the notes from the referring hospital there was no record of administration of phenothiazines or related neuroleptic drugs. Two weeks after the fall, the patient was transferred to our care. He was conscious and oriented; anarthria was present, with oromandibular involuntary movements consisting of forceful jaw opening, closure and lateral deviation along with facial distortion of varying periods and irregular sequence. There was almost continuous severe bilateral blephrospasm, except for momentary ability to open the eyes every few minutes. The tongue also exhibited abnormal attitudes. Phonation was short, hoarse and of low amplitude. The patient could not masticate and swallow food. Attempts at speaking, swallowing and getting up accentuated the involuntary movements which were less at rest and subsided during sleep. There was retrocollis and mild extensor rigidity in all limbs with superimposed athetotic movements. The limbs could not be used in any functional manner and walking was not possible. The patient could communicate only by brief gestures and phonations. The rest of the clinical neurological examination was normal. During the three week's stay in hospital the patient continued to have polydipsia and polyurea (output 51 per day).

Overnight water deprivation failed to increase urinary osmolality above $290 \mathrm{mosm} / 1$ in the presence of plasma osmolality of $406 \mathrm{mosm} / \mathrm{l}$. Twenty four hour urinary sodium excretion was $100 \mathrm{~mol} / \mathrm{l}$ and potassium $7 \cdot 1 \mathrm{mmol} / \mathrm{l}$. Investigations with normal results included haemoglobin, white cell count, blood sugar, blood urea, serum sodium and potassium, CSF protein, sugar and cells. EEG was borderline abnormal with slight excess of $6-7 \mathrm{~Hz}$ theta waves bilaterally diffusely. Unenhanced CT scan revealed a high attenuation lesion with values equivalent to blood in the region of the right globus pallidus (fig).

Carbamazepine $200 \mathrm{mg}$ three times a day was prescribed for the diabetes insipidus. The axial and appendicular dystonia improved rapidly during hospitalisation. CT scan two weeks later showed nearly total resolution of the haematoma. Thirty days after the onset, he was able to walk a few steps without support. Blephrospasm and oromandibular dystonia were also reduced.

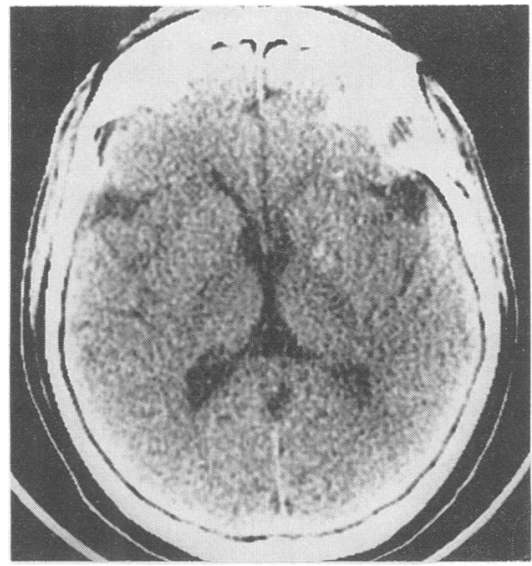

Fig Unenhanced CT scan showing high attenuation lesion in right basal ganglia.

A follow up examination two months later revealed complete disappearance of movement disorder. Urine output ranged between 2.5 to 3.51 per day with specific gravity between 1008 to 1014 .

If the definition of Meige's syndrome includes strict criteria of non-reversibility or progression and idiopathic causation then our patient can not be labelled as suffering from the disease. But the more important issue is the occurrence of movement disorder simulating Meige's disease in association with haematoma in right basal ganglia. The temporal sequence of sudden appearance of movement disorder after head injury and the simultaneous resolution of haematoma and amelioration of clinical deficit strongly implicate the lesion in the right globus pallidus as the cause of the involuntary movements. Dystonia of axial and appendicular muscles as seen in our patient is a known though relatively uncommon feature of Meige disease. ${ }^{13}$

There are very few reports in the literature of pathological or radiological evidence of organic lesion in patients with Meige disease and blephrospasm..$^{\text {7-9 }}$ Unilateral or bilateral basal ganglia infarction ${ }^{78}$ and rostral brain stem lesions in the vicinity of diencephalon" have been reported in patients with blephrospasm. Hemidystonia has been described as a late progressive complication due to focal basal ganglia lesion after mild head trauma. ${ }^{10}$ Partial amelioration of the clinical deficit has been reported in a few cases. ${ }^{34}$ The unusual recovery of the disorder as observed in our patient can be explained by resolution of a small haematoma leaving behind minimal damage to brain parenchyma.
While posttraumatic diabetes insipidus is a well described, though uncommon entity, ${ }^{11}$ its concurrence with a movement disorder supposedly due to dopaminergic preponderance $^{12}$ is of interest in the light of knowledge about neuroendocrine relationship of the posterior pituitary with the hypothalamus and other brain regions. ${ }^{13} 14$ Hypothalamic dopaminergic neurons have rich connections to the median eminence. ${ }^{13}$ The afferent connections of neurohypophysial neurons are of diverse nature and include many extrahypothalamic regions. Neurotransmitters active upon neurohypophysial neurons include dopamine, apart from acetylcholine and norepinephrine. Dopamine has an inhibitory influence upon the posterior pituitary ${ }^{14}$ and hence a disease state with overaction of that system may lead not only to Meiges syndrome, but also by suppression of vasopressin secretion, to diabetes insipidus.

The authors acknowledge the secretarial assistance of Miss Bimla.

APOORVA PAURANIK ASHOK VERMA

MC MAHESHWARI

Department of Neurology Neurosciences Centrē All India Institute of Medical Sciences New Delhis 음

References

1 Marsden CD. Blephrospasm-Oromandibula dystonia syndrome (Brueghel's Syndrome) J Neurol Neurosurg Psychiatry 1976;39: 1201-9.

2 Paulson GW. Meiges syndrome. Geriatrics 1972;27:69-73

3 Tolosa ES. Clinical features of Meige's disease (Idiopathic orofacial dystonia). A report of 17 cases. Arch Neurol 1981;38:147-51.

4 Gollomp SM, Fahn S, Burke RE, Reches A Ilson J. Therapeutic trials in Meige syndrome. Adv Neurol 1983:37:207-13.

5 Garcia-Albea E, Franch O, Munoz D, Ricoy JR. Brueghel's syndrome, report of a case with postmortem studies. J Neurol Neurosurg Psychiatry 1981;44:437-40.

6 Forno LS. Reaction of the substantia nigra to massive basal ganglia infarction (Case 10) Acta Neuropathol (Berl) 1983;62:96-102.

7 Powers JM. Blephrospasm due to unilatera diencephalon infarction (Letter). Neurology 1985;35:283-4.

8 Keane JR, Young JA. Blepharospasm with bilateral basal ganglia infarction. Arch Neurol 1985;42:1206-8.

9 Jankovic J, Patel SC. Blephrospasm associated with brainstem lesions. Neurology 1983;33:1237-40. 
10 Brett EM, Hoarse RD, Sheehy MP, Marsden CD. Progressive hemidystonia due to focal basal ganglia lesion after mild head trauma. J Neurol Neurosurg Psychiatry 1981;44:460.

11 Randall RV, Clark EC, Dodge HW, Bahn RC, Bulbulian AH. Diabetes Insipidus: current concepts in the production of Anti-diuretic hormone: clinical and experimental observations. Post Grad Med 1961;29:102.

12 Tolosa ES. Lai Chi wan: Meige disease: Striatal dopaminergic preponderance. Neurology 1979;29:1126-30.

13 Palkovits M. Topography of chemically identified neurons in the central nervous system. Progress in 1981-1983. In: Muller EE, Macleod RM, eds. Neuroendocrine Perspectives. Amsterdam: Elsevier 1984:8.

14 Brown GM, Friend WC, Chambers JW. Neuropharmacology of hypothalamic pituitary regulation. In: Tolis G, Martin JB, Labrie F, Naftolin F, eds. Clinical Neuroendocrinology. A Pathophysiological Approach. New York: Raven Press, 1979:54 and 69-70.

Accepted 15 August 1986

\section{Anaplastic astrocytoma associated with human growth hormone administration}

Sir: Laron and Josefsberg ${ }^{1}$ reported two deaths out of 166 children treated with human growth hormone (HGH). One of these died from a large cystic brain tumour shown on angiography; however necropsy was not performed and no histological diagnosis made. They called for further reports of neurological disease and deaths encountered in other patients on HGH therapy.

In Glasgow, an 18 year old male who had been treated with HGH for seven years, later developed an astrocytoma. Only 35 cases of astrocytoma have been recorded in the West of Scotland between 1974-1983 in the 15-25 year age-group (personal communication. West of Scotland Cancer Surveillance Unit). This patient was started on HGH therapy at age 9 years in 1977, in a dose of 4 I.U. three times weekly until 1984. In 1986 he presented with a ten day history of headache, malaise, nausea and vomiting. On examination he had papilloedema, and a computer tomography brain scan revealed a large cystic tumour deep in his right parietal lobe. Major craniotomy was not recommended as this was liable to cause severe neurological deficit, and a limited biopsy procedure was performed, revealing anaplastic astrocytoma on histology. He deteriorated rapidly and died.
Natural HGH has been withdrawn in both UK and USA because of its association with Creutzfeldt-Jacob disease, ${ }^{2}$ but association with astrocytoma has not previously been described.

JAMES EG O'NEIL

Stobhill General Hospital Glasgow, G21 3UW, UK

\section{References}

1 Laron $Z$, Josefsberg $Z$. Withdrawal of human pituitary growth hormone. Lancet 1985;i: 1324.

2 Milner RDG. Growth Hormone 1985. Br Med J 1985;291:1593-4.

\section{Skull base chondroma presenting in preg- nancy}

Sir: We present the case of a patient with multiple chondromatosis and rapidly evolving cranial nerve palsies during two successive pregnancies. No skull lesion had been apparent previously. Symptomatic cure followed partial excision of a chondroma arising from the skull base. A subsequent pregnancy proceeded uneventfully to term.

A 29 year old woman complained of left facial numbness and pain after 5 weeks amenorrhoea. Three weeks later she developed a partial left ptosis which became complete after another week.

At the age of 14 she had noticed swellings on the hands and feet, several of which required curettage for cosmetic reasons or because of local trauma. These were benign enchondromata. Asymptomatic lesions were also apparent radiologically in the limbs and in several ribs. Her first pregnancy at the age of $21 \mathrm{yr}$ had been complicated by preeclampsia but ended in normal delivery of a healthy baby and the second pregnancy had been terminated "thera-peutically" in the first trimester. Fifteen months before this admission she had complained of numbness of the left side of her face after 8 weeks amenorrhoea; this resolved following a spontaneous abortion. No neurological symptoms occurred during her normal menstrual cycle.

Examination revealed scars, localised hard swellings over several long bones and deformity of the left forearm. There was a complete left third nerve palsy and she had reduced sensation in the ophthalmic territory of the left trigeminal nerve, a reduced corneal reflex, anaesthesia of the two lower divisions and an ipsilateral trigeminal motor palsy. Skull radiographs, CT scan and carê otid angiography revealed an enlarged for amen ovale, irregularity of the petrous bonec expansion of the clivus and dorsum sellae, soft tissue mass in the posterios nasopharynx and narrowing and displace ment of the internal carotid artery.

Despite the well established benign nature of the limb lesions urgent exploration of the cranial lesion was felt to be indicated as it rapid presentation and progression raise the possibility of malignancy. The preg nancy was terminated on medical advice? (with some reduction in facial pain) and one week later craniotomy was performedo revealing a pinkish-grey tumour extendin $\overline{\bar{g}}$. into the cavernous sinus and through whict the third, fourth, fifth and sixth craniaf nerves passed. An extensive internal decomes pression of the partly mucinous tumour was undertaken. Histology confirmed a benign chondroma with no features of malignancy $\vec{\omega}$ there was cellular enlargement and a swollew matrix.

Partial resolution of the third nerve palsy occurred within 3 days and there was für return of sensation in the two lower trigento inal divisions by one week. Further recowiry took place and after 9 months the on residual complaint was of infrequent paras esthesiae of the left cheek without abnormal signs. One year later she expreßsed a wish to become pregnant again, provo[ing mixed reactions from her medical adviser The consensus was in favour of pregnatis before a natural increase in cell numbers and residual tumour bulk could again cease symptoms. Since that time she had ${ }^{+} a b$ uncomplicated pregnancy, delivering healthy baby.

Chondromata of the skull are uncommon Arising from synchondroses, they are slow growing, often solitary and usually benign An increased tendency to undergo malig nant change had been suggested when the occur in patients with multiple chon $\overrightarrow{5}$ dromatosis (Ollier's disease, dysa chondroplasia). ${ }^{1}$ Lesions in the skull base usually present with progressive cranif nerve palsies, while those in the vault max act as mass lesions. ${ }^{2-6}$ They are said to be more common in females. ${ }^{7}$ The rapid progression of our patient's symptoms wa we believe, due to tumour enlargement under the influences of pregnancy. This is $\mathrm{a} B$ established feature of intracranial tumours and most histological types can behave is this way, the commonest being astrocytoma? meningioma and neurofibroma. ${ }^{8}$

The mechanism causing rapid enlarge ment of tumours in pregnancy is the subjege of debate. Although the physiological 